\title{
Adults with Prader-Willi syndrome exhibit a unique microbiota profile
}

\author{
Wendy J. Dahl ${ }^{*}{ }^{*} \mathbb{C}$, Jérémie Auger ${ }^{2}$, Zainab Alyousif ${ }^{1}$, Jennifer L. Miller ${ }^{3}$ and Thomas A. Tompkins²
}

\begin{abstract}
Objective: Adults with Prader-Willi syndrome (PWS) require less energy intake to maintain body weight than the general adult population. This, combined with their altered gastrointestinal transit time, may impact microbiota composition. The aim of the study was to determine if the fecal microbiota composition of adults with PWS differed from non-affected adults. Using usual diet/non-interventional samples, fecal microbiota composition was analyzed using 16S rRNA gene amplicon sequencing and data from adults with PWS were merged with four other adult cohorts that differed by geographical location and age. QIIME $2^{\mathrm{TM}}$ sample-classifier, machine learning algorithms were used to cross-train the samples and predict from which dataset the taxonomic profiles belong. Taxa that most distinguished between all datasets were extracted and a visual inspection of the R library PiratePlots was performed to select the taxa that differed in abundance specific to PWS.

Results: Fecal microbiota composition of adults with PWS showed low Blautia and enhanced RF39 (phyla Tenericutes), Ruminococcaceae, Alistipes, Erysipelotrichacaea, Parabacteriodes and Odoribacter. Higher abundance of Tenericutes, in particular, may be a signature characteristic of the PWS microbiota although its relationship, if any, to metabolic health is not yet known.
\end{abstract}

Keywords: Prader-Willi syndrome, RF39, Blautia, Tenericutes, Microbiota, 165 rRNA

\section{Introduction}

Prader-Willi syndrome (PWS) is a genetic disorder characterized by lower energy requirement, lack of satiety, and hyperphagia, which together lead to obesity if food intake is not strictly controlled [1]. Although reported total fiber intake of adults with PWS $[2,3]$ is similar to the general population [4], constipation is common in this patient population [5]. Microbiota composition may be altered by constipation; the evidence suggests decreased Bifidobacterium [6, 7], Lactobacillus [7] and Bacteroides [6] compared to healthy controls. Further, individuals with constipation-predominant irritable bowel syndrome (IBS) are also reported to have lower Bifidobacterium and Lactobacillus spp., as well as lower Roseburia-E. rectale

\footnotetext{
*Correspondence: wdahl@ufl.edu

${ }^{1}$ Department of Food Science and Human Nutrition, University of Florida,

359 Newell Drive, Gainesville, FL 32611, USA

Full list of author information is available at the end of the article
}

taxa and higher sulfate-reducers [8]. However, in women, fecal microbiota profile was not associated with constipation, but to colonic transit time [9]. Adults with PWS display delayed mean intestinal transit time compared to healthy controls [5], suggesting motility issues which may impact microbiota profile.

In adults with PWS, the microbiota composition has been shown to differ from controls matched for age, gender, and body fat mass index. Specifically, those with PWS had a higher abundance of Akkermansia, Desulfovibrio and taxa of Tenericutes and Archaea, but a lower abundance of Dorea [10]. However, the microbiota composition of these subjects with PWS did not differ significantly from that of their parents. It remains unclear as to whether the microbiota composition of individuals with PWS is characteristic of the syndrome or the environment. Given the interconnections between microbiota, its metabolism and metabolic health, insight into the microbiota profile of PWS, considered a model of 
hyperphagia [11], is of interest. The aim of this exploratory analysis was to determine if the microbiota composition of adults with PWS differed from unaffected adults, independent of geographical location and age.

\section{Main text \\ Methods}

De-identified $16 \mathrm{~S}$ profiles from usual diet/non-interventional fecal samples of 25 adults $(34.9 \pm 10.2$ years; $60 \%$ female) with genetically confirmed PWS and residing in Florida $[3,12]$ were compared to those of healthy adults residing in Canada $(n=151 ; 35.2 \pm 10.1$ years; $61 \%$ female) [13], adults with IBS residing in Canada $(n=263$; $41.8 \pm 15.2 ; 79 \%$ female) [14], healthy young adults residing in Florida, USA ( $n=68 ; 23.2 \pm 3.5 ; 63 \%$ female) [15], and healthy older women residing in Florida $(n=26$; $73.7 \pm 5.6$ years) [16]. All source data originated from the same lab environment and were subsequently treated with the same bioinformatics processing. Details of DNA extraction $[3,16]$ and methods for community-wide taxonomic profiling via $16 \mathrm{~S}$ amplicon sequencing of the fecal samples, carried out in the source studies, were previously reported [3], i.e. no DNA extraction or sequencing was conducted for the purposes of the present analysis. The Institutional Review Board of the University of Florida approved this analysis of de-identified $16 \mathrm{~S}$ profiles as exempt. The data in fastq format were imported into one QIIME artefact (demux.qza) [17]. All reads were quality filtered with same parameters and trimmed at $240 \mathrm{bp}$ on the forward read. Using the Deblur denoiser (implemented as a QIIME 2 module), the amplicon sequence variant (ASV) abundance tables and representative sequences were generated. The representative sequences were merged to make the taxonomic profiles using the 'taxonomy.qza' trained on the GreenGenes database. The ASVs from the abundance table were attributed to known taxonomic names and compiled at the genus level for further analyses.

Using QIIME's visualization tools, the principal coordinates analysis (PCoA), weighted UniFrac, and individual taxonomic profiles were generated and examined [1736]. Group differences were highlighted using QIIME 2 sample-classifier. This module allowed machine learning algorithms to cross-train on the samples and predict the label (here the label is 'from which dataset does the taxonomic profile comes from'). The important features used by the algorithm (those taxa that distinguish the most between dataset) were extracted and the taxa list was used for further exploration. Each important feature was plotted using the $\mathrm{R}$ library PiratePlot ( $\mathrm{R}$ version 3.5.3). Since this taxa list was not made to single out PWS, but rather distinguish between all datasets, a visual inspection of the plots was performed to select the taxa that differed in abundance specific to PWS. Eight PWS-specific taxa appeared in the top 36 important features.

\section{Results}

The Weighted UniFrac PCoA from QIIME of the merged datasets was visualized using Emperor and color coded according to the sample's clinical trial provenance and is shown in Fig. 1. Each data point represents a fecal sample and the distance matrix between them based on

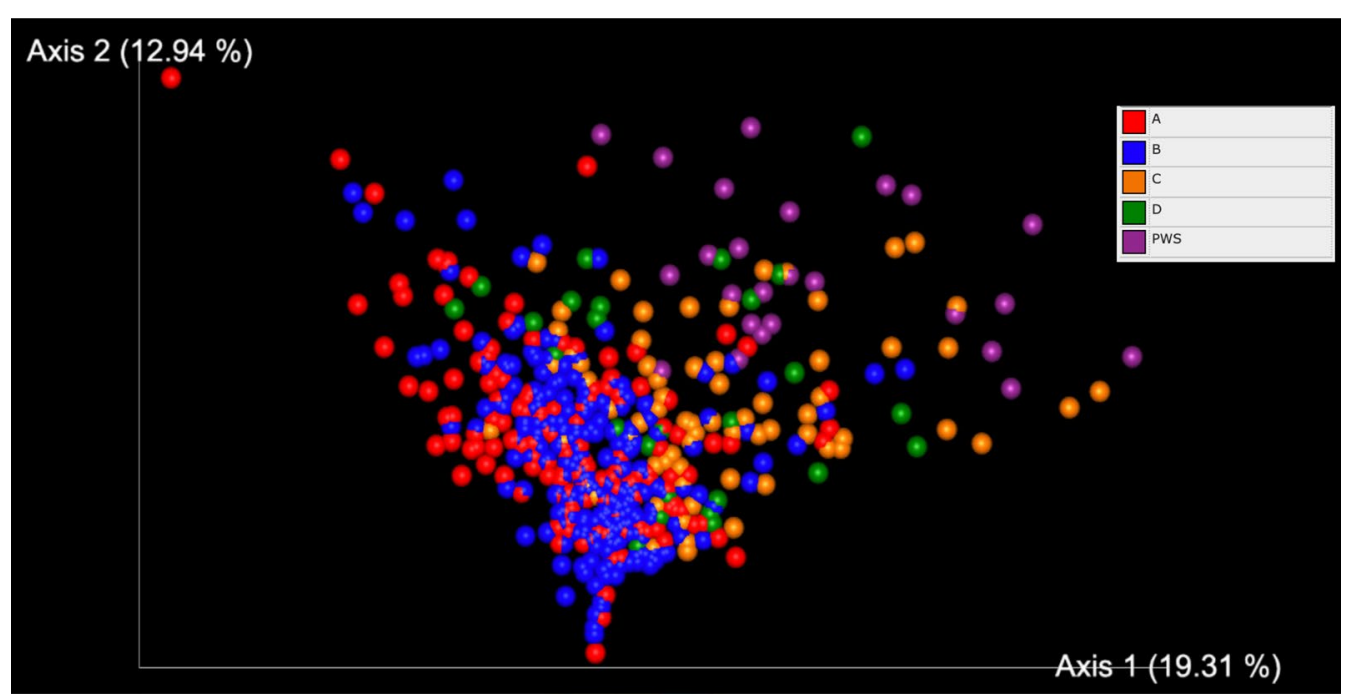

Fig. 1 Beta diversity by Weighted UniFrac Principal Coordinates Analysis (PCoA) of the fecal microbiome data sets of adults with Prader-Willi syndrome (PWS) and (A) adults residing in Canada; (B) adults with irritable bowel syndrome residing in Canada, (C) adults residing in Florida, USA, (D) older women residing in Florida 
ASV counts and phylogeny. The PWS samples seem to cluster (upper right portion) and the other samples all show significant overlap indicates that the PWS profiles are different from those of the other adults. Conversely, PWS profiles have similarities not shared by the other groups. On this basis, a machine learning algorithm was implemented to classify the profiles on sample origin and highlight the most explicative taxa. Figure 2 shows the Machine Learning model-accuracy results for the Extra-Tree classification trained on the merged dataset samples taken at baseline. The confusion matrix shows strong accuracy scores on the main diagonal and a good overall accuracy result of $74.8 \%$ and an accuracy of $100 \%$ for the PWS predictions alone. The training was done on an $80-20 \%$ training-to-testing cross validation ratio. QIIME's sample classifier module heatmap visualization is shown in Fig. 2, depicting the relative abundances of the important taxa used for the classification for each of the groups compared.

The list of important taxa (and associated importance score) was obtained from the 'feature_importance.qza' file generated by the classifier and used for individual taxa plotted in Additional file 1: Fig. S1. Note that the model optimized for overall performance and thus, many of the 36 important features taxa shown are used by the model to distinguish between non-PWS samples. The selection of PWS explaining taxa by manual visualization (taxa graphs where the PWS is obviously different from all the others) suggests 8 taxa of the 36 important features are linked with PWS microbiota profiles (Fig. 3). From the 8 PWS-specific taxa, only the genus Blautia (ranked 1) was lower in PWS than other cohorts. This is of interest given Blautia was the most abundant genus in all datasets, averaging $21 \%$ of the total sample composition. Ruminococcaceae appeared twice (rank 8 and 12) at different taxonomic levels. The genus Alistipes (family Rikenellaceae, phylum Bacteroidetes) ranked 9 and is clearly higher in PWS and nearly absent in some others. Erysipelotrichacaea, Parabacteroides (family Porphyromonadaceae) and Odoribacter, ranked 17, 18 and 33, respectively, also appeared higher in the PWS dataset. Finally, rank 15 was the order RF39 (phylum Tenericutes, class Mollicutes) and it seems to be strongly linked with PWS, as the maximum values of relative abundances for this taxon in samples of non-affected adult cohorts are much lower than those of PWS. Of note, rank 3 (Additional file 1: Fig. S1)

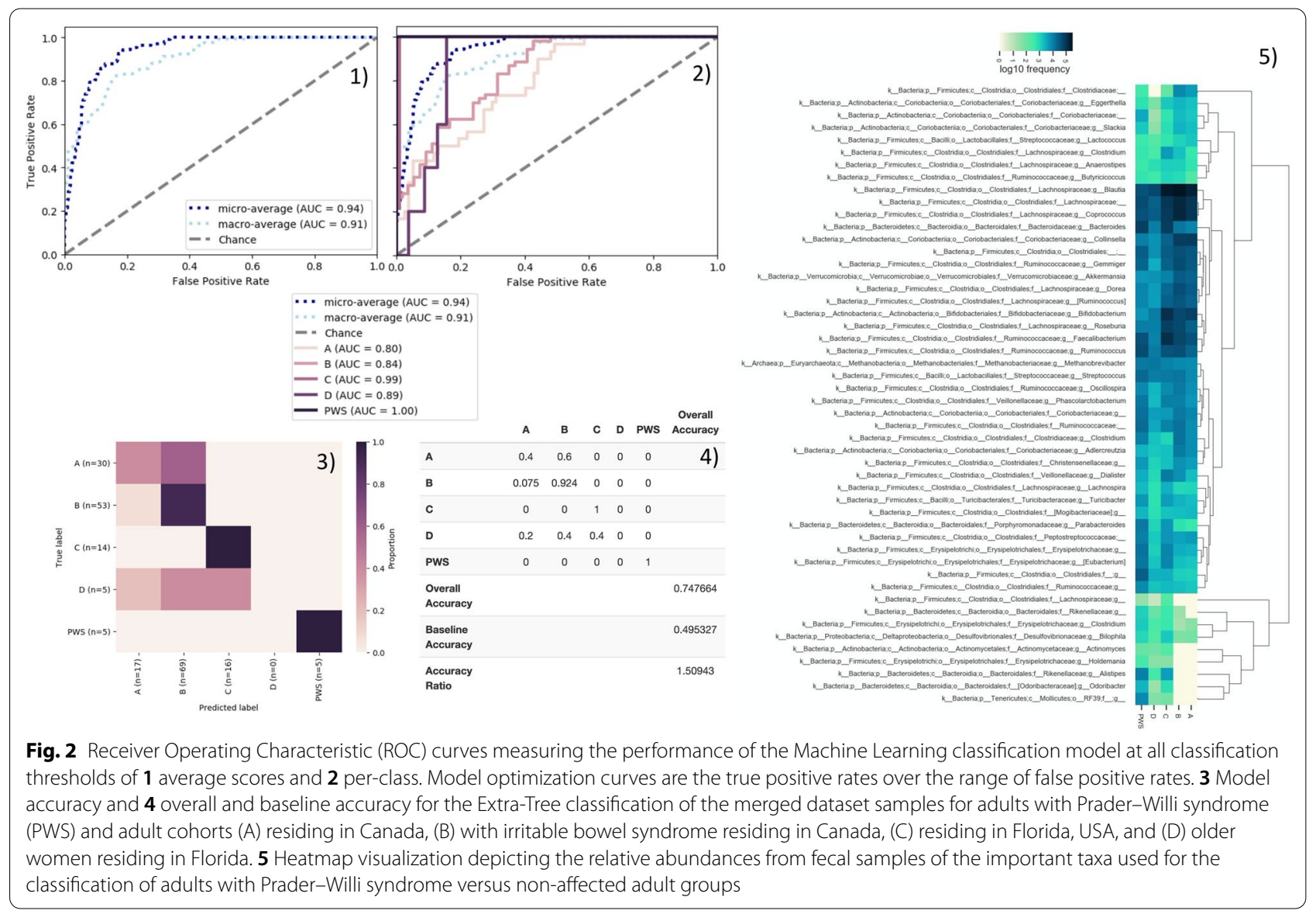



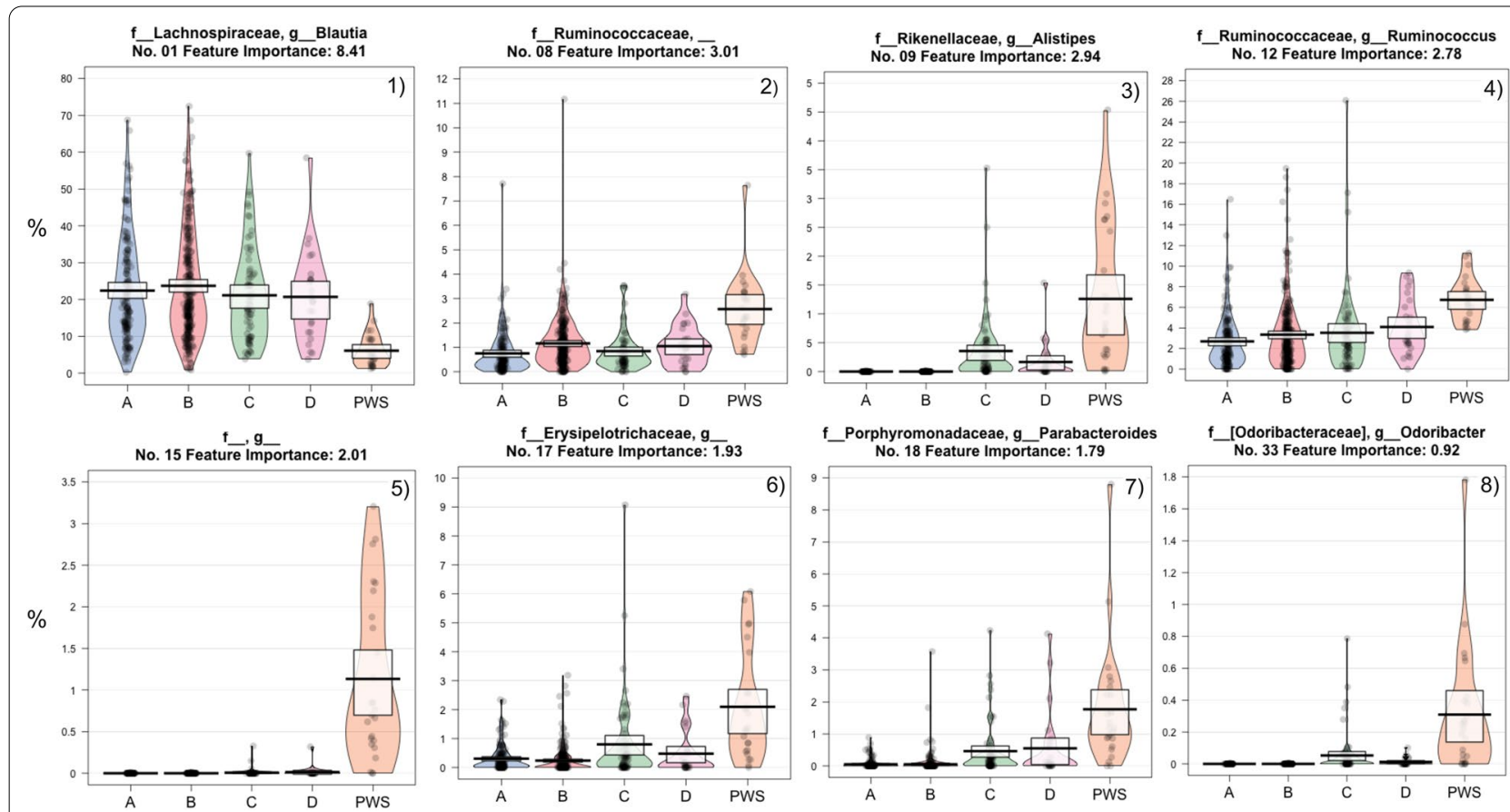

Fig. 3 PiratePlots of the relative abundances of 8 taxa, specifically $\mathbf{1}$ genus Blautia, $\mathbf{2}$ family Ruminococcaceae, $\mathbf{3}$ genus Alistipes $\mathbf{4}$ genus Ruminococcus, $\mathbf{5}$ order RF39 $\mathbf{6}$ family Erysipelotrichaceae $\mathbf{7}$ genus Parabacteroides, and $\mathbf{8}$ genus Oridobacter, which by manual, visual inspection, represent apparent differences between the Prader-Willi syndrome (PWS) microbiota profiles compared to adults (A) residing in Canada, (B) adults with irritable bowel syndrome residing in Canada, (C) adults residing in Florida, USA, and (D) older women residing in Florida

suggests Bifidobacterium abundance of the PWS profiles were similar to the older women but lower than the other adult cohorts.

\section{Discussion}

Previous research suggests that adults with PWS may harbor a microbiota composition with unique characteristics [10]. In the present study, the abundance of Bifidobacterium was lower in PWS, with the exception of the older women who exhibited similarly low levels. This finding was not unexpected given that suppression of Bifidobacterium has been reported in individuals with constipation [7] and some of the adults with PWS reported slow transit stool form, suggestive of constipation [3]. This finding is concerning though given the protective role of Bifidobacterium spp. in maintaining intestinal eubiosis and barrier function [37]. PWS adults exhibited higher abundance of Tenericutes (order RF39), Alistipes, Parabacteroides, and Odoribacter, as well as Ruminococcaceae and Erysipelotrichaceae, compared to the non-affected adults. Of interest, RF39 was one of the taxa identified by Olssen et al. in PWS adults [10] and by Peng et al. in children with PWS compared to matched controls [38]. The relevance of higher levels of Tenericutes in PWS adults is not known; however, the abundance of Alistipes and Parabacteroides have been negatively associated with cardiometabolic indices such as serum lipids, blood glucose, and blood pressure [39]. Additionally, Ruminococcaceae has been negatively associated with metabolic syndrome [40] and lower long-term weight gain [41]. Conversely, abundance of Erysipelotrichaceae has been associated with obesity and lipid metabolism, and specific taxa within this family may be inflammatory and immunogenic [42]. Both Odoribacter and Alistipes have been associated with diet quality [43]. Olsson et al. showed that adults with PWS had lower abundance of Dorea compared to obese controls [10]. Similarly, Peng et al., by random forest analyses, also identified a difference in Dorea in children with PWS when compared to controls [38]. Dorea was not an identifying taxon for PWS in the present study. However, the adults with PWS profiled in this analysis had much lower body mass index (BMI) [3] than the subjects in the Olsson study. Lower abundance of Blautia in adults with PWS presents as an interesting enigma. Three genera in the Lachnospiraceae family have been shown to be positively correlated with BMI, namely Blautia, Dorea, and Ruminococcus [44]. Blautia abundance has been shown to be inversely associated with visceral fat area-after adjustment for age, BMI, and other lifestyle-related factors [45]. Adults with PWS present with lower visceral fat area compared to healthy adults [46], which is thought to 
contribute to their reduced risk of developing type 2 diabetes [47]. Thus, given their weight status and typically lower visceral fat, it might be expected that individuals with PWS would have higher abundance of Blautia. Of note, Blautia abundance also has been correlated with higher serum insulin and impaired lipid metabolism [48], suggesting a benefit for low abundance. The finding of lower levels of Blautia in adults with PWS may, therefore, correlate with their relative insulin sensitivity despite frequent obesity. In summary, the microbiota profile findings may suggest benefit related to the reported cardiometabolic protection in PWS [47].

Blautia spp. utilize dietary carbohydrates [49], thus the restricted carbohydrate intake of the PWS adults [3] may have contributed to lower Blautia abundance. Lower abundance of Blautia has been shown in athletes consuming a higher protein, lower carbohydrate diet, compared to sedentary controls [50]. Although the absolute intake of protein, fat and carbohydrate of the adults with PWS is a fraction of the intake of athletes, the percentage of energy from protein was similar [3], and thus macronutrient composition may contribute to Blautia abundance. However, no association between protein, fat, carbohydrate or fiber intake with Blautia abundance was found in a large cross-sectional study [45]. Of further interest is the relationship between Blautia abundance and gastrointestinal symptoms. IBS patients, whose gastrointestinal symptoms decreased with a low-FODMAP diet, had higher abundance of Blautia [51], suggesting visceral sensitivity in these individuals. In contrast, individuals with PWS exhibit a high tolerance to pain and discomfort [52]. The adults with PWS who provided fecal samples for this analysis reported minimal gastrointestinal discomfort [3], similar to healthy individuals $[15,53]$. The possibility of a relationship between Blautia abundance and visceral sensitivity requires further investigation.

The results of this analysis provide further evidence that the microbiota composition of individuals with PWS differs from that of unaffected individuals, notably with the presence of higher Tenericutes, specifically the order RF39, although the implications to health are unknown. Further, it may be interesting to explore the relationship between Blautia abundance and visceral sensitivity, as well as metabolic health, in PWS and other patient populations. Given their low prevalence of Bifidobacterium spp., the PWS population may benefit from synbiotic supplementation.

\section{Limitations}

This study had limitations. Foremost, the study was undertaken as a post hoc analysis. Its major limitation was the use of a merged dataset to undertake the comparison of PWS to non-affected adults, as the fecal samples were not processed in the same batch. However, all samples were all processed using exactly the same methodology in terms of collection, storage, sequencing, and bioinformatics. A single database, Greengenes, was used; results may differ if data was analyzed using another database such as SILVA.

\section{Supplementary Information}

The online version contains supplementary material available at https://doi. org/10.1186/s13104-021-05470-6.

Additional file 1: Figure S1. PiratePlots of all taxa distinguishing groups of the merged datasets including: (A) adults residing in Canada; (B) adults with irritable bowel syndrome residing in Canada, (C) adults residing in Florida, USA D) older women residing in Florida, and PWS) adults with Prader-Willi syndrome.

\section{Abbreviations}

BMI: Body mass index; IBS: Irritable bowel syndrome; PCoA: Principal coordinates analysis; PWS: Prader-Willi syndrome.

\section{Acknowledgements}

None.

\section{Authors' contributions}

ZA, WJD and JM conducted the primary study which included the PWS fecal collections. JA, TAT and WJD designed the analysis. JA and TAT analyzed the data. WJD and JA wrote the manuscript. All authors read and approved the final manuscript.

\section{Funding}

Lallemand Health Solutions Inc. funded this analysis. TAT and JA, employed by funder, were involved in the design of the analysis, interpretation of data, and writing the manuscript. ZA was the recipient of a doctoral scholarship from the King Abdullah Scholarship Program, Saudi Arabian Cultural Mission (SACM).

\section{Availability of data and materials}

The dataset of the PWS adults is available at: https://www.ncbi.nlm.nih.gov/ bioproject/?term=PRJNA669563. The full dataset generated and analyzed during the current study is not publicly available but deidentified data are available from the corresponding author upon reasonable request.

\section{Ethics approval and consent to participate}

The study protocol was approved by the Institutional Review Board of the University of Florida and deemed non-human research of de-identified samples and exempt (IRB202002813), and thus informed consent was not applicable.

\section{Consent for publication}

Not applicable.

\section{Competing interests}

JA and TAT are employees of the Rosell Institute for Microbiome and Probiotics, the research group of Lallemand Health Solutions Inc., the funder of this analysis. WJD, ZA and JLM declare no conflict of interest.

\section{Author details}

1 Department of Food Science and Human Nutrition, University of Florida, 359 Newell Drive, Gainesville, FL 32611, USA. ${ }^{2}$ Rosell Institute for Microbiome and Probiotics, 6100 Royalmount, Montreal, QC H4P 2R2, Canada. ${ }^{3}$ Division of Endocrinology, Department of Pediatrics, College of Medicine, University of Florida, 1600 SW Archer Road, Gainesville, FL 32610, USA. 
Received: 5 November 2020 Accepted: 28 January 2021

Published online: 06 February 2021

\section{References}

1. Butler MG, Miller JL, Forster JL. Prader-Willi syndrome-clinical genetics, diagnosis and treatment approaches: an update. Curr Pediatr Rev 2019;15(4):207-44. https://doi.org/10.2174/15733963156661907161 20925.

2. Woods SG, Knehans A, Arnold S, Dionne C, Hoffman L, Turner P, et al. The associations between diet and physical activity with body composition and walking a timed distance in adults with Prader-Willi syndrome. Food Nutr Res. 2018. https://doi.org/10.29219/fnr.v62.1343.

3. Alyousif Z, Miller JL, Auger J, Sandoval M, Piano A, Tompkins TA, et al. Microbiota profile and efficacy of probiotic supplementation on laxation in adults affected by Prader-Willi syndrome: a randomized, double-blind, crossover trial. Mol Genet Genom Med. 2020. https://doi.org/10.1002/ mgg3.1535.

4. Reicks M, Jonnalagadda S, Albertson AM, Joshi N. Total dietary fiber intakes in the US population are related to whole grain consumption: results from the National Health and Nutrition Examination Survey 2009 to 2010. Nutr Res. 2014;34(3):226-34. https://doi.org/10.1016/j.nutre s.2014.01.002

5. Kuhlmann L, Joensson IM, Froekjaer JB, Krogh K, Farholt S. A descriptive study of colorectal function in adults with Prader-Willi syndrome: high prevalence of constipation. BMC Gastroenterol. 2014;14:63. https://doi. org/10.1186/1471-230X-14-63.

6. Kim SE, Choi SC, Park KS, Park MI, Shin JE, Lee TH, et al. Change of fecal flora and effectiveness of the short-term VSL\#3 probiotic treatment in patients with functional constipation. J Neurogastroenterol Motil. 2015;21(1):111-20. https://doi.org/10.5056/jnm14048.

7. Khalif IL, Quigley EM, Konovitch EA, Maximova ID. Alterations in the colonic flora and intestinal permeability and evidence of immune activation in chronic constipation. Dig Liver Dis. 2005;37(11):838-49. https:// doi.org/10.1016/j.dld.2005.06.008.

8. Chassard C, Dapoigny M, Scott KP, Crouzet L, Del'homme C, Marquet $\mathrm{P}$, et al. Functional dysbiosis within the gut microbiota of patients with constipated-irritable bowel syndrome. Aliment Pharmacol Ther. 2012;35(7):828-38. https://doi.org/10.1111/j.1365-2036.2012.05007.x.

9. Parthasarathy G, Chen J, Chen X, Chia N, O'Connor HM, Wolf PG, et al. Relationship between microbiota of the colonic mucosa vs feces and symptoms, colonic transit, and methane production in female patients with chronic constipation. Gastroenterology. 2016;150(2):367.e1-379.e1. https://doi.org/10.1053/j.gastro.2015.10.005.

10. Olsson LM, Poitou C, Tremaroli V, Coupaye M, Aron-Wisnewsky J, Backhed F, et al. Gut microbiota of obese subjects with Prader-Willi syndrome is linked to metabolic health. Gut. 2019. https://doi.org/10.1136/gutjn I-2019-319322.

11. Tauber M, Diene G, Mimoun E, Çabal-Berthoumieu S, Mantoulan C, Molinas $C$, et al. Prader-Willi syndrome as a model of human hyperphagia. Front Horm Res. 2014;42:93-106. https://doi.org/10.1159/000358317.

12. Alyousif Z, Miller JL, Sandoval MY, MacPherson CW, Nagulesapillai V, Dahl WJ. The effects of Bifidobacterium animalis ssp. lactis B94 on gastrointestinal wellness in adults with Prader-Willi syndrome: study protocol for a randomized controlled trial. Trials. 2018;19(1):256. https://doi. org/10.1186/s13063-018-2648-x

13. MacPherson CW, Mathieu O, Tremblay J, Champagne J, Nantel A, Girard $\mathrm{SA}$, et al. Gut bacterial microbiota and its resistome rapidly recover to basal state levels after short-term amoxicillin-clavulanic acid treatment in healthy adults. Sci Rep. 2018;8(1):11192. https://doi.org/10.1038/s4159 8-018-29229-5.

14. Lewis ED, Antony JM, Crowley DC, Piano A, Bhardwaj R, Tompkins TA, et al. Efficacy of Lactobacillus paracasei HA-196 and Bifidobacterium longum R0175 in alleviating symptoms of irritable bowel syndrome (IBS): a randomized placebo-controlled study. Nutrients. 2020. https://doi. org/10.3390/nu12041159.

15. Tremblay A, Fatani A, Ford AL, Piano A, Nagulesapillai V Auger J, et al. Safety and effect of a low- and high-dose multi-strain probiotic supplement on microbiota in a general adult population: a randomized, double-blind, placebo-controlled study. J Diet Suppl. 2020. https://doi. org/10.1080/19390211.2020.1749751.

16. Ford AL, Nagulesapillai V, Piano A, Auger J, Girard SA, Christman M, et al. Microbiota stability and gastrointestinal tolerance in response to a high protein diet with and without a prebiotic, probiotic and synbiotic: a randomized, double-blind, placebo-controlled trial in older women. J Acad Nutr Diet. 2020:120(4):500-16. https://doi.org/10.1016/j.jand.2019.12.009.

17. Bolyen E, Rideout JR, Dillon MR, Bokulich NA, Abnet CC, Al-Ghalith GA, et al. Reproducible, interactive, scalable and extensible microbiome data science using QIIME 2. Nat Biotechnol. 2019;37(8):852-7. https://doi. org/10.1038/s41587-019-0209-9.

18. Bokulich NA, Subramanian S, Faith JJ, Gevers D, Gordon JI, Knight R, et al. Quality-filtering vastly improves diversity estimates from Illumina amplicon sequencing. Nat Methods. 2013;10(1):57-9. https://doi.org/10.1038/ nmeth.2276.

19. Bolyen E, Rideout JR, Dillon MR, Bokulich NA, Abnet CC, Al-Ghalith GA, et al. QIIME 2: reproducible, interactive, scalable, and extensible microbiome data science. PeerJ Preprints. 2018. https://doi.org/10.1038/s4158 7-019-0209-9.

20. Vázquez-Baeza Y, Pirrung M, Gonzalez A, Knight R. EMPeror: a tool for visualizing high-throughput microbial community data. Gigascience. 2013;2(1):16. https://doi.org/10.1186/2047-217X-2-16.

21. Vázquez-Baeza Y, Gonzalez A, Smarr L, McDonald D, Morton JT, NavasMolina JA, et al. Bringing the dynamic microbiome to life with animations. Cell Host Microbe. 2017;21(1):7-10. https://doi.org/10.1016/j. chom.2016.12.009.

22. McDonald D, Clemente JC, Kuczynski J, Rideout JR, Stombaugh J, Wendel $D$, et al. The biological observation matrix (BIOM) format or: how I learned to stop worrying and love the ome-ome. Gigascience. 2012;1(1):7. https ://doi.org/10.1186/2047-217X-1-7.

23. Katoh K, Standley DM. MAFFT multiple sequence alignment software version 7: improvements in performance and usability. Mol Biol Evol. 2013;30(4):772-80. https://doi.org/10.1093/molbev/mst010.

24. Lozupone C, Knight R. UniFrac: a new phylogenetic method for comparing microbial communities. Appl Environ Microbiol. 2005;71(12):8228-35. https://doi.org/10.1128/AEM.71.12.8228-8235.2005.

25. Lozupone CA, Hamady M, Kelley ST, Knight R. Quantitative and qualitative beta diversity measures lead to different insights into factors that structure microbial communities. Appl Environ Microbiol. 2007;73(5):1576-85. https://doi.org/10.1128/AEM.01996-06.

26. Chang Q, Luan Y, Sun F. Variance adjusted weighted UniFrac: a powerful beta diversity measure for comparing communities based on phylogeny. BMC Bioinform. 2011;12:118. https://doi.org/10.1186/1471-2105-12-118.

27. Chen J, Bittinger K, Charlson ES, Hoffmann C, Lewis J, Wu GD, et al. Associating microbiome composition with environmental covariates using generalized UniFrac distances. Bioinformatics. 2012;28(16):2106-13. https ://doi.org/10.1093/bioinformatics/bts342.

28. McDonald D, Vázquez-Baeza Y, Koslicki D, McClelland J, Reeve N, Xu Z, et al. Striped UniFrac: enabling microbiome analysis at unprecedented scale. Nat Methods. 2018;15(11):847-8. https://doi.org/10.1038/s4159 2-018-0187-8.

29. Weiss S, Xu ZZ, Peddada S, Amir A, Bittinger K, Gonzalez A, et al. Normalization and microbial differential abundance strategies depend upon data characteristics. Microbiome. 2017;5(1):27. https://doi.org/10.1186/s4016 8-017-0237-y.

30. Amir A, McDonald D, Navas-Molina JA, Kopylova E, Morton JT, Xu ZZ, et al. Deblur rapidly resolves single-nucleotide community sequence patterns. mSystems. 2017. https://doi.org/10.1128/mSystems.00191-16.

31. Stackebrandt E, Goodfellow M. Nucleic acid techniques in bacterial systematics. Chichester:Wiley; 1991.

32. Caporaso JG, Kuczynski J, Stombaugh J, Bittinger K, Bushman FD, Costello EK, et al. QIIME allows analysis of high-throughput community sequencing data. Nat Methods. 2010;7(5):335-6. https://doi.org/10.1038/nmeth f.303.

33. McKinney W. Data structures for statistical computing in python. In: Proceedings of the 9th Python in science conference, Austin, TX; 2010.

34. Legendre P, Legendre LF. Numerical ecology. Amsterdam: Elsevier; 2012.

35. Halko N, Martinsson P-G, Shkolnisky Y, Tygert M. An algorithm for the principal component analysis of large data sets. SIAM J Sci Comput. 2011:33(5):2580-94. 
36. Price MN, Dehal PS, Arkin AP. FastTree 2-approximately maximum-likelihood trees for large alignments. PLoS ONE. 2010. https://doi.org/10.1371/ journal.pone.0009490.

37. lacob S, lacob DG. Infectious threats, the intestinal barrier, and its Trojan horse: dysbiosis. Front Microbiol. 2019;10:1676. https://doi.org/10.3389/ fmicb.2019.01676.

38. Peng Y, Tan Q, Afhami S, Deehan EC, Liang S, Gantz M, et al. The gut microbiota profile in children with Prader-Willi syndrome. Genes. 2020. https://doi.org/10.3390/genes11080904.

39. Zeng Q, Li D, He Y, Li Y, Yang Z, Zhao X, et al. Discrepant gut microbiota markers for the classification of obesity-related metabolic abnormalities. Sci Rep. 2019;9(1):13424. https://doi.org/10.1038/s41598-019-49462-w.

40. He Y, Wu W, Wu S, Zheng HM, Li P, Sheng HF, et al. Linking gut microbiota, metabolic syndrome and economic status based on a population-level analysis. Microbiome. 2018;6(1):172. https://doi.org/10.1186/s4016 8-018-0557-6.

41. Menni C, Jackson MA, Pallister T, Steves CJ, Spector TD, Valdes AM. Gut microbiome diversity and high-fibre intake are related to lower long-term weight gain. Int J Obes. 2017;41(7):1099-105. https://doi.org/10.1038/ ijo.2017.66.

42. Kaakoush NO. Insights into the role of Erysipelotrichaceae in the human host. Front Cell Infect Microbiol. 2015;5:84. https://doi.org/10.3389/fcimb 2015.00084

43. Liu Y, Ajami NJ, El-Serag HB, Hair C, Graham DY, White DL, et al. Dietary quality and the colonic mucosa-associated gut microbiome in humans. Am J Clin Nutr. 2019;110(3):701-12. https://doi.org/10.1093/ajcn/nqz139.

44. Ottosson F, Brunkwall L, Ericson U, Nilsson PM, Almgren P, Fernandez C, et al. Connection between BMI-related plasma metabolite profile and gut microbiota. J Clin Endocrinol Metab. 2018;103(4):1491-501. https://doi. org/10.1210/jc.2017-02114.

45. Ozato N, Saito S, Yamaguchi T, Katashima M, Tokuda I, Sawada K, et al. Blautia genus associated with visceral fat accumulation in adults 20-76 years of age. NPJ Biofilms Microbiomes. 2019;5:28. https://doi. org/10.1038/s41522-019-0101-x

46. Sode-Carlsen R, Farholt S, Rabben KF, Bollerslev J, Schreiner T, Jurik AG, et al. Body composition, endocrine and metabolic profiles in adults with
Prader-Willi syndrome. Growth Horm IGF Res. 2010;20(3):179-84. https:// doi.org/10.1016/j.ghir.2009.12.004.

47. Crino A, Grugni G. Update on diabetes mellitus and glucose metabolism alterations in Prader-Willi syndrome. Curr Diab Rep. 2020;20(2):7. https:// doi.org/10.1007/s11892-020-1284-5.

48. Lippert K, Kedenko L, Antonielli L, Kedenko I, Gemeier C, Leitner M, et al. Gut microbiota dysbiosis associated with glucose metabolism disorders and the metabolic syndrome in older adults. Benef Microbes. 2017:8(4):545-56. https://doi.org/10.3920/BM2016.0184.

49. Oliphant K, Allen-Vercoe E. Macronutrient metabolism by the human gut microbiome: major fermentation by-products and their impact on host health. Microbiome. 2019;7(1):91. https://doi.org/10.1186/s4016 8-019-0704-8.

50. Jang LG, Choi G, Kim SW, Kim BY, Lee S, Park H. The combination of sport and sport-specific diet is associated with characteristics of gut microbiota: an observational study. J Int Soc Sports Nutr. 2019;16(1):21. https:// doi.org/10.1186/s12970-019-0290-y.

51. Laatikainen R, Jalanka J, Loponen J, Hongisto SM, Hillila M, Koskenpato J, et al. Randomised clinical trial: effect of low-FODMAP rye bread versus regular rye bread on the intestinal microbiota of irritable bowel syndrome patients: association with individual symptom variation. BMC Nutr. 2019;5:12. https://doi.org/10.1186/s40795-019-0278-7.

52. Priano L, Miscio G, Grugni G, Milano E, Baudo S, Sellitti L, et al. On the origin of sensory impairment and altered pain perception in Prader-Willi syndrome: a neurophysiological study. Eur J Pain. 2009;13(8):829-35. https://doi.org/10.1016/j.ejpain.2008.09.011.

53. Hanifi A, Culpepper T, Mai V, Anand A, Ford A, Ukhanova M, et al. Evaluation of Bacillus subtilis R0179 on gastrointestinal viability and general wellness: a randomised, double-blind, placebo-controlled trial in healthy adults. Benef Microbes. 2014;6(1):19-27. https://doi.org/10.3920/BM201 4.0031

\section{Publisher's Note}

Springer Nature remains neutral with regard to jurisdictional claims in published maps and institutional affiliations.
Ready to submit your research? Choose BMC and benefit from:

- fast, convenient online submission

- thorough peer review by experienced researchers in your field

- rapid publication on acceptance

- support for research data, including large and complex data types

- gold Open Access which fosters wider collaboration and increased citations

- maximum visibility for your research: over $100 \mathrm{M}$ website views per year

At $\mathrm{BMC}$, research is always in progress.

Learn more biomedcentral.com/submissions 